\title{
Study on the Joint Pressure of Forward and Backward Walking and Its
}

\section{Mechanism}

\author{
Peng Song ${ }^{1}$, Tingran Zhang ${ }^{2}$, Jiong Luo ${ }^{2}$ \\ ${ }^{1}$ Faculty of Materials and Energy, Southwest University, Beibei, Chongqing, 400715 \\ ${ }^{2}$ College of Physical Education .Southwest University, Beibei, Chongqing, 400715
}

KEYWORDS: Join Pressure; Forward and Backward Walking; Function Mechanism

\begin{abstract}
Walking in the current world has become one of the most popular sports items, walking motion easiest, most convenient and most economical, therefore, in the past few decades, in pursuit of a healthy lifestyle, the number of people walking increases every year, more and more people choose to exercise by walking movement and body weight control. Research on the forward and backward walking joint pressure changes in this paper, experimental methods, the results show that the forward and backward traveling support phase PFJCF peak difference depends on the size of the ground reaction force, ground reaction force vector relative knee position and lower limb link angular acceleration. At lower speeds, PFJCF peak difference was not significant, however, does not exclude the presence of difference between the two at higher speeds, the future need to be further explored at different speeds forward and backward walking support phase PFJCF peak difference.
\end{abstract}

\section{Introduction}

Walking is the ability of human life freedom of action essential, through the interaction of lower limb bones, muscles, joints and nervous system, the moving body can be arbitrary. Everyday ordinary people engaged in various activities in order to make the body, you need to make a lot of lower limb movements, which related lower limb movements due to the different needs of various actions constituted, can be divided into walking, jogging, running, jumping, sliding the front side glide back and walking seven basic forms. And different forms of lower limb movement, the force may be the case in the vertical direction to 1 times the weight of more than 5 times the weight, for example: the force is minimal walking and walking back, compared with only slightly higher than body weight; and sliding and other actions before sliding the force of about 2 times the weight; jogging, compared with 2.5 times the weight; Run is close to 4 times the weight; and hop is more than five times more weight. For most people, walking forward a simple basic movement, we are also a number of studies the forward walking and Nordic walking on human effectiveness do in-depth discussion, but for a different way of walking backward walking as research is minimal. Walking back, it is a built on the basis of the forward walking a human instinct. In clinical practice, often walking back stroke patients as a gait training or physical treatment, Thomas mentioned in the book, were walking back to avoid lower limb training synergies generated in stroke patients who can enhance selectivity to produce lower limb movement, thereby improving lower limb when walking in a better walking pattern. 


\section{Subjects and Methods}

The study target. Beijing University of Physical Education 8 Health Physical Education college students as subjects, the basic situation: age (21.50 \pm 0.53$)$ years, height $(172.2 \pm 3.89) \mathrm{cm}$, weight $(63.2 \pm 3.72) \mathrm{kg}$. Subjects no recent knee injuries and pain and have no professional training to walk back. Before the test, described in detail the purpose of the experiment, precautions and possible problems, the subject written informed consent, in accordance with the test requirements with voluntary staff test.

Research Methods. Subjects were asked on a $10 \mathrm{~m}$ long runway forward and backward walking speed is $10 \mathrm{~km} / \mathrm{h}$ (equivalent to $2.77 \mathrm{~m} / \mathrm{s}$ ). Finnish Photocells speedometer set two infrared light emitting device testing walking speed, Photocells speedometer with built-in average walking speed infrared light measurement subject through verbal feedback so that all participants have reached the same target speed $10 \mathrm{~km} / \mathrm{h}$, to the nearest target speed test run data as research. Before the test, the first through lectures and demonstrations on the action, then all subjects were given plenty of exercise to be confident to walk back. Kinematic and kinetic data using motion capture and the US Motion Analysis System acquisition, through eight Raptor-4 Digital camera motion capture (Motion, USA) collecting kinematic data acquisition frequency of $200 \mathrm{~Hz}$, using inverse dynamics analysis software configuration MOTION data, using double-sided adhesive reflective signs point directly affixed to the skin, Motion flag stickers reflective dot placed reference model uses Helen Hayes, using Kistler force plates collected ground reaction force data acquisition frequency of 1000 $\mathrm{Hz}$, walking along the direction is the positive direction of the $\mathrm{X}$-axis, $\mathrm{Y}$-axis in the horizontal plane and perpendicular to the $\mathrm{X}$-axis points to the left, Z-axis positive direction vertically upwards.

Calculation and data processing. It is defined as the angle between the knee of the thigh and lower leg coordinate system coordinates Euler angles of rotation about the $\mathrm{X}$ axis first, to obtain flexion angle. Quadriceps tendon force $(\mathrm{Fq})$ calculated by the following formula: $\mathrm{Fq}=\mathrm{Mk} / 0.049 \mathrm{~m}$, which, Fq is the quadriceps tendon force, Mk knee muscle torque; PFJCF (FPFJC) mainly through the quadriceps tendon forces and the relationship between the quadriceps tendon and patellar tendon angle between the calculated primarily by the following formula: FPFJC $=2 \mathrm{~F} \alpha \cdot \sin (\beta / 2)$, where the patellar tendon and quadriceps tendon angle $\beta$ is based on the relationship between knee angle and structure of the patellofemoral angle between the calculated. PFJCF peak (N) refers to the forward or backward walking support phase, the maximum PFJCF absolute; PFJCF relative peak (BW) is to eliminate the differences in body weight affect PFJCF peak, at the same time, the influence of gravity incorporated into the calculation of the relative peak PFJCF be dimensionless treatment, with a relative peak of PFJCF weight ratio normalized to do, namely: PFJCF relative peak $(\mathrm{BW})=$ PFJCF peak $(\mathrm{N}) \div 9.8(\mathrm{~N} / \mathrm{kg}) \div$ body weight $(\mathrm{kg})$, wherein, BW is not a physical unit name, only with the weight ratio of the amount of peak power table; knee muscle peak torque (Nm) refers to the forward or backward walking support phase, the human knee suffered Rally and the product of the peak tension arm; knee muscle relative peak torque $(\mathrm{Nm} / \mathrm{kg})$ in order to eliminate the differences in body weight on knee muscle peak torque, with peak torque divided by the weight of the knee muscle, namely: knee muscle torque relative peak $(\mathrm{Nm} / \mathrm{kg})=$ knee muscle peak torque $(\mathrm{Nm}) \div$ body weight $(\mathrm{kg}))$; instant touchdown and knee angle from an instant to mean an extension cord to connect the hip and knee and knee with the connection between the angle of the ankle joint; knee flexion and extension range is defined in the forward or backward walking support phase, the knee angle difference between the maximum and minimum values between. Kinematics and dynamics of all variables through statistical indicators for regular and Spss19.0 Origin8.0 software, test data interpolation and normalization, then averaged (x) of each index and standard deviation (s ), the significance level of 0.05 , the forward and backward walking PFJCF peak (N), PFJCF relative 
peak (BW), knee muscle peak torque $(\mathrm{Nm})$, knee muscle relative peak torque $(\mathrm{Nm} / \mathrm{kg})$, touch an instant knee angle $\left(^{\circ}\right)$, from an instant knee angle $\left(^{\circ}\right)$, knee flexion and extension range $\left(^{\circ}\right.$ ) and other indicators of differences in test paired sample Wilcoxon signed rank test (Wilcoxon signed-rank test).

\section{Results and Analysis}

Walking forward support phase PFJCF peak (2 $280.68 \pm 296.36$ ) N, walking back support phase PFJCF peak (2 $011.57 \pm 597.32) \mathrm{N}$, although the forward walking than walking back support phase PFJCF peak large, but the two groups PFJCF peak statistics found that the forward and backward walking PFJCF peak difference was not significant $(\mathrm{P}=0.208)$; in order to eliminate the effect of body weight on the measurement results, and further calculates the relative peak PFJCF found that walking forward support phase PFJCF relative peak was $3.57 \pm 0.43$, walking back support phase PFJCF relative peak $3.14 \pm 0.92$, walk forward support phase PFJCF relative peak still support phase PFJCF relative peak greater than walking back, but the difference between the two is not significant $(\mathrm{P}=0.208)$; walk forward knee muscle peak torque $(136.87 \pm 16.35) \mathrm{Nm}$, walking back knee muscle peak torque $(114.40 \pm 28.02) \mathrm{Nm}$, walking forward knee muscle peak torque than the back to walk knee muscle peak torque large, but the difference between the two is not significant ( $\mathrm{P}$ $=0.091)$; walk forward knee muscular torque relative value $(2.10 \pm 0.27) \mathrm{Nm} / \mathrm{kg}$, running back knee muscle torque relative value $(1.75 \pm 0.41) \mathrm{Nm} / \mathrm{kg}$, walking forward knee muscular torque relative value than walking back knee muscle torque relative value is greater, but the difference between the two is not significant $(\mathrm{P}=0.063)$; forward walking knee touch the ground instantaneous angle $(6.13 \pm 3.21)^{\circ}$, walking back knee angle $(6.13 \pm 3.21)^{\circ}$, the difference was significant $(\mathrm{P}=0.012)$; walk forward from an instant knee angle $(13.16 \pm 6.67)^{\circ}$, walking back knee angle $(6.73 \pm 3.89)^{\circ}$, the difference was significant $(\mathrm{P}=0.036)$; knee range of forward travel $(29.15 \pm 4.31)^{\circ}$, walking back to $(33.73 \pm 5.40)^{\circ}$, the difference was not significant $(\mathrm{P}=0.093)$.

\section{In The Impact of Speed of Forward and Backward Traveling on the Joint Pressure Peaks}

Walking forward and back support phase PFJCF peak point of view is not uniform, may be due to inconsistent researchers selected walking speed. In fact, with the increase in walking speed, impact force peak, the second peak in the vertical direction, the braking force peak, peak acceleration and direction of the force of the three-dimensional fluctuation range also increases, that may impact on the speed peak PFJCF. Sussman DH compared 10 subjects at $1.0 \mathrm{~m} / \mathrm{s}$ speed of forward and backward walking supporting phase PFJCF size found at this speed, walking back and forward during walking and knee extensor PFJCF torque difference was not statistically significant, at slower speeds, forward and backward traveling exhibit similar PFJCF; in our study on the basis of Sussman DH on the forward and backward walking speed increased to $2.78 \mathrm{~m} \cdot \mathrm{s}-1$, at the rate study found that the forward and backward walking is still showing a similar PFJCF; however, Flynn's study, subjects forward and backward are self-selected walking speed suitable speed , resulting in a positive phase PFJCF walking support relative peak of about $5.6 \pm 1.3$, walking back support phase PFJCF relative peak of about $3.0 \pm 0.6$; Scott and Winter calculated at $3.5 \sim 5.3 \mathrm{~m} \bullet$ s-1 positive rate walking to support phase PFJCF relative peak of about 7.0 to 11.1. Thus, the speed has a great impact on the results of PFJCF. At lower speeds, forward and backward walking support PFJCF peak phase difference may not be significant, however, does not exclude the presence of difference between the two at higher speeds, the future need to be further explored at a higher speed the forward and backward traveling support phase difference PFJCF peak. 


\section{Conclusion}

Walking forward and back when walking the opposite hip rotation after the knee, kick joint angle displacement characteristics are very similar, but the two moves articulation range FF mode performance for significantly less than the walk back to walk forward; normal gait walking FF, the walk back in pace, cadence, stride length was significantly shorter than the other aspects of walking forward, step time, double FF hold time, the first double support time was significantly longer; and walk forward compared to walk back support during ankle dorsiflexion angle maximum FF significantly increased, and the maximum swing angle of plantar flexion, knees before the maximum swing angle, swing angle of maximum flexion FF, the support of the largest extension cord angle and heel when riding off hip flexion angle was significantly reduced.

\section{Acknowledgements}

Fund Project: Central Universities Fundamental Research Projects Funded Project" Biomechanical Mechanisms of Forward and Backward Walking and Its Enhancement Effect of Physical Fitness.

Project No.:XDJK2016C056

\section{REFERENCE:}

[1] Thomas M A, Fast A. One step forward and two steps back: The dangers of walking backwards in therapy [J]. American Journal of Physical Medicine \& Rehabilitation, 2001,79 (8): 459-461.

[2] Y R Yang, R Y Wang, L L Yen Lieu Gait outcomes after additional backward walking training in patients with strok [J]. a randomized controlled trial Clinical Rehabilitation, 19 (2005), pp: 264-273.

[3] M.E. Hackney, G M. Earhart Tai Chi improves balance and mobility in people with Parkinson disease [J]. Gait and Posture, 28 (2008), pp. 456-460.

[4] R. Grasso, L Bianchi F. Lacquaniti Motor patterns for human gait backward versus forward locomotion [J]. Journal of Neurophysiology, 80 (1998), pp. 1868-1885.

[5] A Thorstensson How is the normal locomotor program modified to produce backward walking [J]. Experimental Brain Research, 61 (1996), pp: 664-668.

[6] Malhotra, Singh S.The beneficial effect of yoga in diabetes [J]. Nepal Medical College, 2005,7 (2): 145-147. 